\title{
e-Phaïstos
}

e-Phaïstos

Revue d'histoire des techniques / Journal of the history

of technology

IX-2 | 2021

Quel objet pour quel musée?

\section{Réflexion sur la conservation et la valorisation du patrimoine scientifique et technique}

L'objet scientifique et technique au prisme de l'expérience muséale

Bringing Technical and Industrial Cultural Heritage closer to the Public

Odile Lassère

\section{(2) OpenEdition}

Journals

Édition électronique

URL : https://journals.openedition.org/ephaistos/9598

DOI : 10.4000/ephaistos.9598

ISSN : 2552-0741

Éditeur

IHMC - Institut d'histoire moderne et contemporaine (UMR 8066)

Référence électronique

Odile Lassère, « Réflexion sur la conservation et la valorisation du patrimoine scientifique et technique », e-Phaïstos [En ligne], IX-2 | 2021, mis en ligne le 26 octobre 2021, consulté le 28 octobre 2021. URL : http://journals.openedition.org/ephaistos/9598; DOI : https://doi.org/10.4000/ephaistos. 9598

Ce document a été généré automatiquement le 28 octobre 2021.

Tous droits réservés 


\section{Réflexion sur la conservation et la valorisation du patrimoine scientifique et technique}

L'objet scientifique et technique au prisme de l'expérience muséale

Bringing Technical and Industrial Cultural Heritage closer to the Public

\section{Odile Lassère}

\section{Avant-propos}

Cet article ouvre des pistes de réflexion sur la conservation et la mise en valeur du patrimoine scientifique, technique et industriel avec, pour cadre de référence, le Code du patrimoine. Rédigé en 2017, l'article permet d'évoquer un outil essentiel de la gestion des musées : le Projet Scientifique et Culturel dont chaque «musée de France » se dote afin de tracer - pour plusieurs années - sa feuille de route. Cet article traite pour partie du PSC du musée de l'Histoire du fer préparé entre 2017 et 2019. En 2021, ce PSC est toujours en discussion. En 2019, le Pôle Muséal de la métropole du Grand Nancy est constitué sous la direction de Pierre-Antoine Gérard, conservateur en chef du patrimoine, à partir du Muséum-Aquarium de Nancy, du musée de l'Histoire du fer et du château de Montaigu. La définition du programme de rénovation du musée de l'Histoire du fer, adossé au PSC, est actuellement réinterrogée par la métropole du Grand Nancy pour l'adapter à l'ambition de développer un projet culturel à l'échelle du territoire métropolitain. Ces éléments de contexte conjugués à cette ambition de politique culturelle métropolitaine expliquent l'évolution de la situation du musée de l'Histoire du fer entre 2017 et 2021. Son Projet scientifique et culturel nécessite aujourd'hui des adaptations et les réflexions se poursuivent quant à la valorisation de ce patrimoine industriel et technique dans le respect du Code du Patrimoine. Cet article livre donc un état de la réflexion à un moment où l'avenir du musée n'est pas stabilisé. 


\section{Réflexion sur la conservation et la valorisation du patrimoine scientifique et technique}

2 L'extrême diversité des objets conservés au musée de l'Histoire du fer, au nombre de près de 14000 , permet d'aborder plusieurs cas de figure de conservation et de mise en valeur des collections scientifiques et techniques. Les fondateurs du musée sont deux passionnés d'archéologie, spécialistes du métal, Édouard Salin (1889-1970) et Albert France-Lanord (1915-1993) et un historien des techniques, Bertrand Gille (1920-1980) ${ }^{1}$. Ce dernier a rassemblé, spécifiquement pour le musée, un grand nombre d'objets techniques et industriels, d'œuvres d'art (arts graphiques, œuvres d'art) en lien avec la métallurgie et la sidérurgie.

3 Établissement de culture scientifique et technique de la métropole du Grand Nancy et de l'Université de Lorraine, le musée de l'Histoire du fer (fig.1) est un «musée de France » dont les collections et l'histoire muséales sont uniques en leurs genres. Il a pour vocation de montrer et de promouvoir l'histoire universelle de la sidérurgie et l'histoire des techniques de l'industrie. L'établissement se caractérise en outre par ses liens avec la restauration des métaux et avec la recherche: il abrite le Laboratoire d'archéologie des métaux, atelier de restauration des métaux, créé en 1950, partie intégrante et originale du projet initial en tant que service à part entière du musée (Folzan 2011).

Fig.1. Le musée de l'Histoire du fer

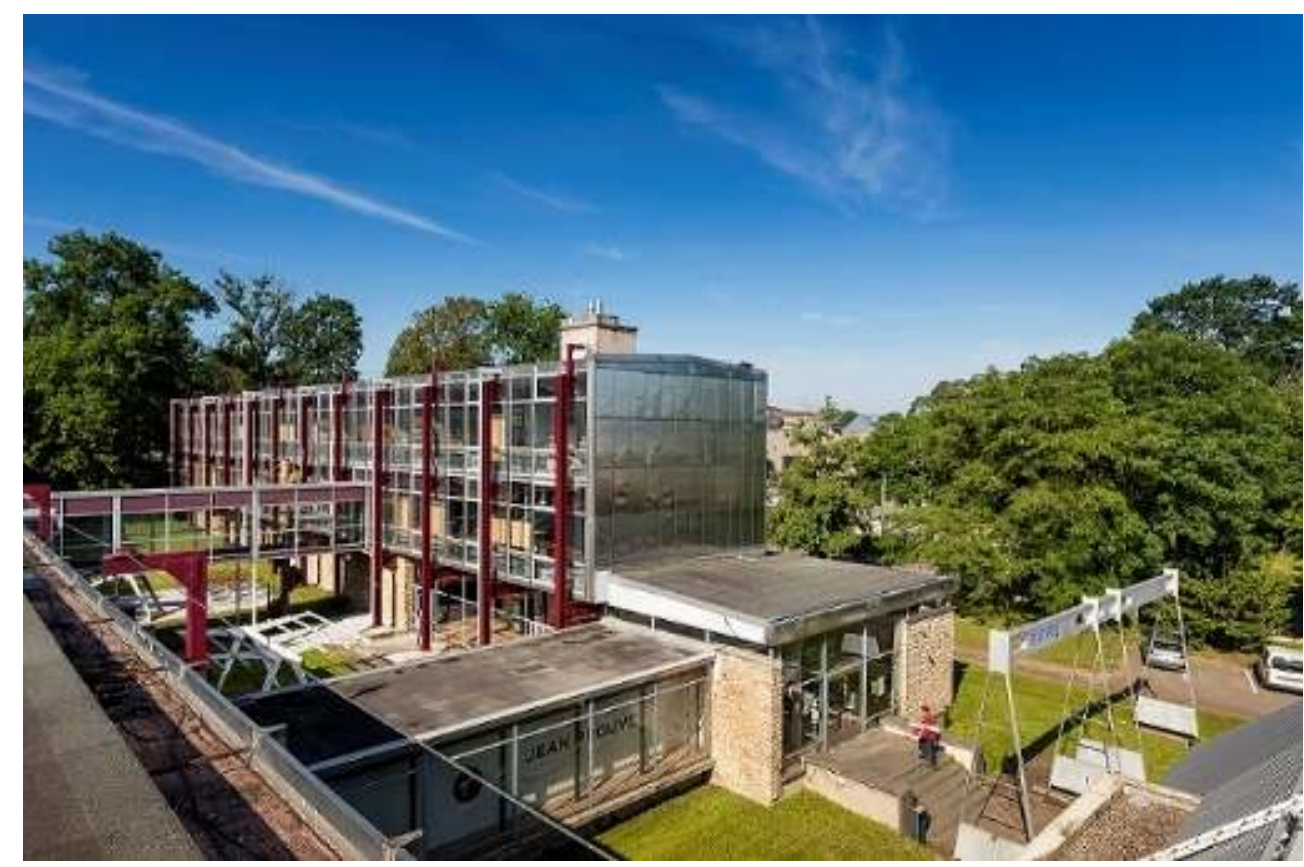

Le bâtiment a été construit par Michel et Jacques André avec Claude Prouvé, entre 1961 et 1966.

(c) Musée de l'Histoire du fer, Jarville-la-Malgrange, cliché Olivier Dancy

Dans une région qui oscille entre désindustrialisation et ré-industrialisation (Raggi 2019), le musée attirait trop peu de public (seulement 10000 visiteurs par an) et ne s'était pas encore suffisamment renouvelé (Lassère 2017) malgré sa situation au cœur de la métropole du Grand Nancy, où des réussites scientifiques et entrepreneuriales, 
des travaux sur les matériaux et les métaux offrent malgré tout de stimulantes perspectives. Le musée a ouvert au public en 1966 ; son équipe et son organisation se sont professionnalisées et structurées selon le cadre de référence fixé par le Code du Patrimoine, la loi sur les Musées de 2002 (Livre IV Musées articles L410 à L452-4). Après cinquante-cinq années d'ouverture au public et la loi sur les Musées de 2002, le musée de l'Histoire du fer a été doté d'un Projet Scientifique et culturel en 2019. Ce travail de réflexion a été l'occasion de faire le point sur la constitution de la collection et sa valorisation pour les publics (Lassère 2016). La réflexion est toujours en cours pour adapter au mieux le musée à son public et à son environnement et le PSC est à nouveau en discussion ${ }^{2}$.

5 La première partie de cet article pose le cadre. Le système français de la loi de 2002 sur les musées de France, aujourd'hui codifiée au livre IV du Code du Patrimoine ${ }^{3}$, place la matérialité des objets et le maintien de leur intégrité matérielle au centre de la conservation du patrimoine. La mise en contexte des objets (nature, contexte de la collecte...) est également un principe essentiel. Les conservateurs et personnels scientifiques en charge des collections s'interrogent en permanence sur l'opportunité de conserver et de faire entrer - ou non - des objets à l'inventaire des collections soumis au Code du Patrimoine ou de les considérer comme simple matériel d'étude. Dans une deuxième partie, nous présentons les spécificités du patrimoine scientifique et technique: richesse, variété, diversité des interprétations des objets techniques, monumentalité mais aussi difficultés de conservation ou de transmission du sens. Enfin, la troisième partie aborde la valorisation des objets techniques. Plusieurs propositions sont faites : révéler l'ensemble des significations de l'objet à travers des présentations scénographiques, relier les objets aux territoires dont ils sont issus, favoriser l'expérimentation par des manipulations, autant de pistes pour que le public devienne acteur de sa visite. Le conservateur du patrimoine s'interroge aussi sur les objets plus contemporains : que conserver d'aujourd'hui pour demain?

\section{L'objet scientifique et technique « patrimonialisé »}

\section{Contexte général en France}

6 Un musée conserve et valorise une collection d'œuvres d'art et d'objets. Sur les 1220 «musées de France », on peut en compter au moins 300 possédant des collections plus ou moins importantes dans le domaine de la «Culture Scientifique, Technique et Industrielle » (Mariani-Ducray 2004), partie intégrante de la culture au sens large défini par l'Association des musées et centres de science pour le développement de la CSTI (AMCSTI) ; « en permettant au citoyen de comprendre le monde dans lequel il vit et de se préparer à vivre dans celui de demain, en développant l'information et la réflexion sur la science et ses enjeux, en favorisant les échanges avec la communauté scientifique, en partageant les savoirs, en éduquant à une citoyenneté active, la CSTI inscrit la science dans la société » (Gillot $2017: 6$ ).

7 Les collections peuvent être très diverses : certains musées conservent des collections homogènes, se référant à un secteur scientifique, technique ou industriel bien précis : par exemple, l'industrie du sel à Salins-les-Bains, la dentelle à Calais, la lunetterie à Morez. D'autres conservent des collections techniques généralistes: le musée des arts et métiers couvre ainsi sept domaines: ceux de l'instrumentation scientifique, des 
matériaux, de la construction, de la communication, de l'énergie, de la mécanique et des transports. Enfin certains musées traitent de l'histoire des techniques, de l'histoire industrielle, tout en relevant, en parallèle, de l'histoire sociale, de l'histoire environnementale, de l'histoire de l'art... Certains musées d'histoire ou de Beaux-arts s'ouvrent également à l'histoire des techniques.

\section{Les missions des « musées de France » et la matérialité de l'objet de musée}

8 Les «musées de France ", régis par la loi de 2002, aujourd'hui codifiée au livre IV du Code du Patrimoine, ont pour mission de conserver, restaurer, étudier et enrichir leurs collections. La loi renvoie à l'objet de collection proprement dit. Ce sont bien des objets que l'on collecte et que l'on valorise en lien avec une thématique ou un secteur pour sauvegarder et valoriser une mémoire matérielle. Les musées ont également pour mission de rendre les collections accessibles au public le plus large, de concevoir et mettre en œuvre des actions d'éducation et de diffusion visant à assurer l'égal accès de tous à la culture.

9 L'inaliénabilité et l'imprescriptibilité des collections des « musées de France ${ }^{4}$ obligent à s'interroger en amont sur l'opportunité de faire entrer, dans les collections d'un musée, tel ou tel objet. L'inaliénabilité signifie que les collections ne peuvent être ni vendues, ni données, ce qui est conforme à la déontologie des musées, dominée par le souci de conservation et de transmission des œuvres. L'imprescriptibilité signifie qu'en cas de perte ou de vol, les œuvres appartenant au domaine public peuvent être récupérées sans limite de temps même si le nouveau propriétaire est de bonne foi. Le métier de conservateur implique donc de s'interroger sur l'objet, sur sa matérialité, sur le sens qu'il a pour le public au sein d'une collection. L'analyse de l'intérêt patrimonial, déterminante pour une entrée en collection, doit toujours être réalisée en fonction de la vocation du musée traduite dans son Projet scientifique et culturel (Joly 2009 ; Joly 2016). Le PSC est aussi une spécificité française : c'est la feuille de route d'un musée. Les collections et le public de l'établissement inspirent directement ce PSC. Comment cette caractéristique française d'un régime patrimonial associant inaliénabilité et imprescriptibilité s'articule-telle avec la spécificité des collections de sciences et techniques ? Comment, pour les conservateurs du patrimoine, opérer le meilleur choix dans la sélection des œuvres patrimoniales?

\section{La démarche de patrimonialisation : les questions à se poser avant de présenter un dossier devant une commission scientifique d'acquisition}

Pour déterminer l'intérêt public, le Code du Patrimoine prévoit l'examen des projets d'acquisitions par des commissions scientifiques ad hoc. Ainsi l'article R.451-2 énonce que « Toute décision d'acquisition, à titre gratuit ou à titre onéreux, est précédée de l'avis de la Commission scientifique régionale des collections des musées de France. Cette commission examine les projets d'acquisition. » Le conservateur défend, devant la commission scientifique, ses projets d'acquisitions sous la forme d'une note 
d'opportunité argumentée, mettant en perspective le choix de l'objet destiné à intégrer la collection. Plusieurs critères entrent en jeu :

- le nécessaire lien entre l'objet ou l'ensemble à acquérir et la vocation du musée qui souhaite faire entrer cet objet dans ses collections. Ces orientations sont décrites dans le PSC ;

- l'authenticité de l'objet ou de l'ensemble ;

- son intérêt patrimonial : est-ce que cet objet est unique? Est-ce qu'il est représentatif d'un type ou modèle ? D'une époque ? D'une industrie ? D'une innovation technique?;

- une appréciation du prix de cet objet ou de l'ensemble ;

- l'état matériel de l'objet ou de l'ensemble.

11 Lorsqu'on s'interroge sur des objets de sciences et sociétés, on doit aussi réfléchir au périmètre de l'objet que l'on collecte: Où commence et où finit l'objet? Peut-on conserver la mémoire des traces matérielles (les objets et les documents) mais aussi préserver les traces immatérielles (les savoir-faire liés à cet objet technique ?). Les débats au sein des commissions sont souvent vifs et enrichissants. Les commissions d'acquisitions restent encore fortement dominées par des représentants de musées des Beaux-arts assez éloignés des objets de sciences et techniques. Pour les convaincre, les arguments avancés doivent faire mouche et aborder plusieurs aspects de l'objet, de son histoire matérielle, de ses commanditaires, de ses fabricants... !

\section{Collection patrimoniale et matériel d'étude}

12 Nous distinguons, dans les musées, les collections labellisées « musées de France » et les objets répertoriés « matériel d'étude » dont seul l'intérêt documentaire est réel. Dans le domaine des sciences et techniques, de nombreux professionnels étant concernés par cette problématique, la note circulaire du 19 juillet $2012^{5}$ définit les contours de la notion de matériel d'étude et sert de guide méthodologique: les objets collectés "matériel d'étude» font alors l'objet d'études scientifiques et patrimoniales complémentaires nécessaires pour leur faire quitter leur simple fonction d'usage et les faire entrer dans une collection patrimoniale.

\section{Quelques exemples récents d'acquisition au musée de l'Histoire du fer}

Des objets entrent régulièrement dans les collections du musée de l'Histoire du fer.

En 2014, le musée a intégré, dans ses collections patrimoniales, deux instruments scientifiques mis au point par Pierre Chevenard (1888-1960), ingénieur et scientifique français, fondateur de la métallurgie de précision prônant une culture de l'union intime entre la recherche et la réalisation industrielle (Duffaut 2012, 2016) : un dilatomètre différentiel à enregistrement mécanique (1926) et un thermo-magnétomètre enregistreur (1932), deux instruments permettant de caractériser les alliages (fig.2 et 3). La collecte ne s'est pas contentée des seuls objets, elle a également permis de recueillir la mémoire orale de deux anciens enseignants de l'Université de Lorraine, mémoire complétée par une documentation détaillée sur les instruments scientifiques et par les ouvrages de Pierre Chevenard. 
Fig.2. Un thermo-magnétomètre enregistreur (1932), Pierre Chevenard

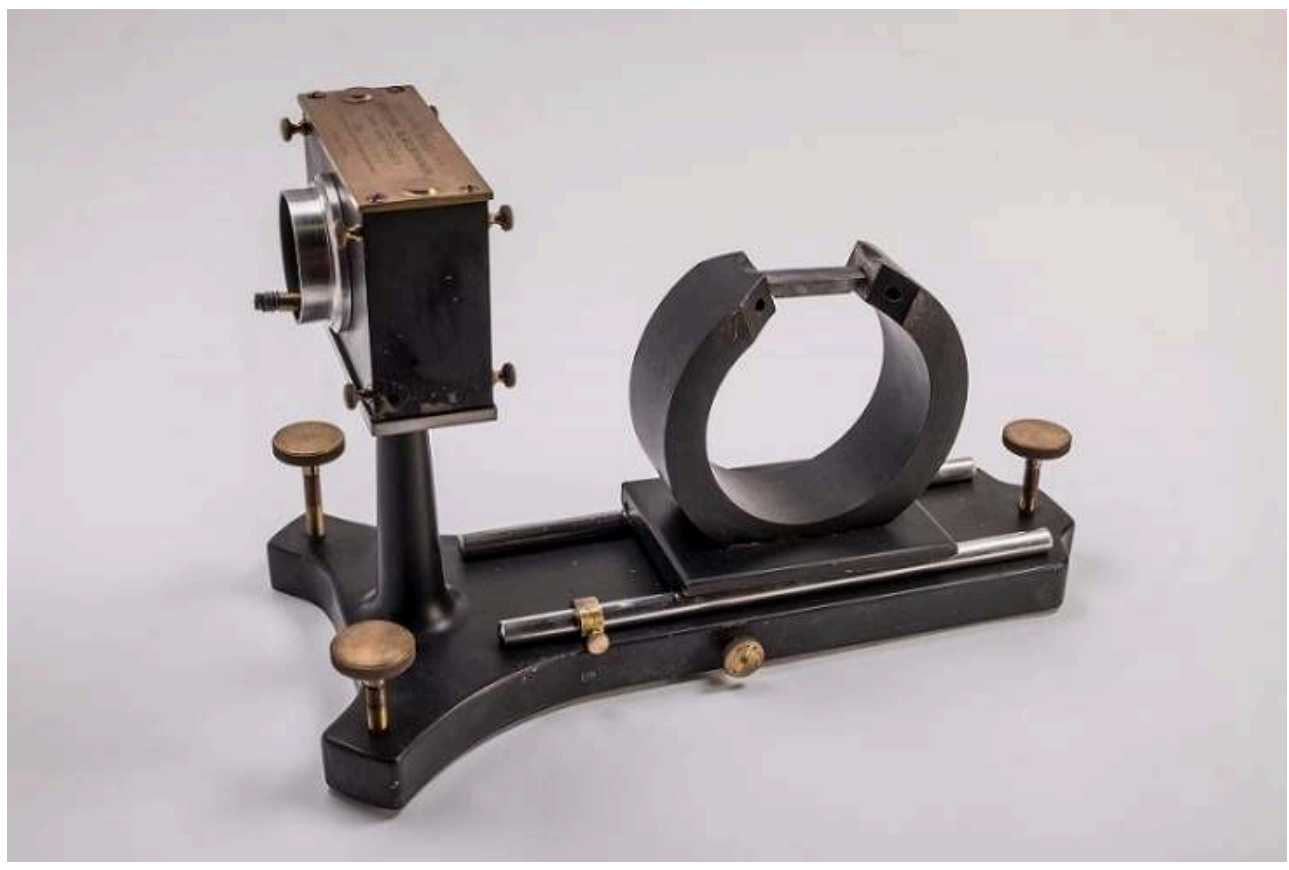

(c) musée de l'Histoire du fer, Jarville-la-Malgrange, cliché Claude Philippot

15 Quel est l'intérêt de ces objets? Ils permettent de raconter une histoire au public : les recherches scientifiques et les résultats appliqués à l'industrie donnent vie aux objets. Ces instruments scientifiques sont importants pour expliquer les qualités des différents métaux, le parcours de Pierre Chevenard et la métallographie adaptée à l'industrie. C'est notamment grâce à des études métallographiques poussées que les ingénieurs de l'entreprise Eiffel ont pu sélectionner la qualité de fer puddlé nécessaire à la construction de la Tour Eiffel. Lors de la fabrication des fers de la tour Eiffel aux fonderies de Pompey au Nord de Nancy, plus de 4200 barrettes de différentes nuances de fers ont été essayées avant réception des livraisons (France-Lanord 1980). 
Fig.3. Un dilatomètre différentiel à enregistrement mécanique (1926), Pierre Chevenard

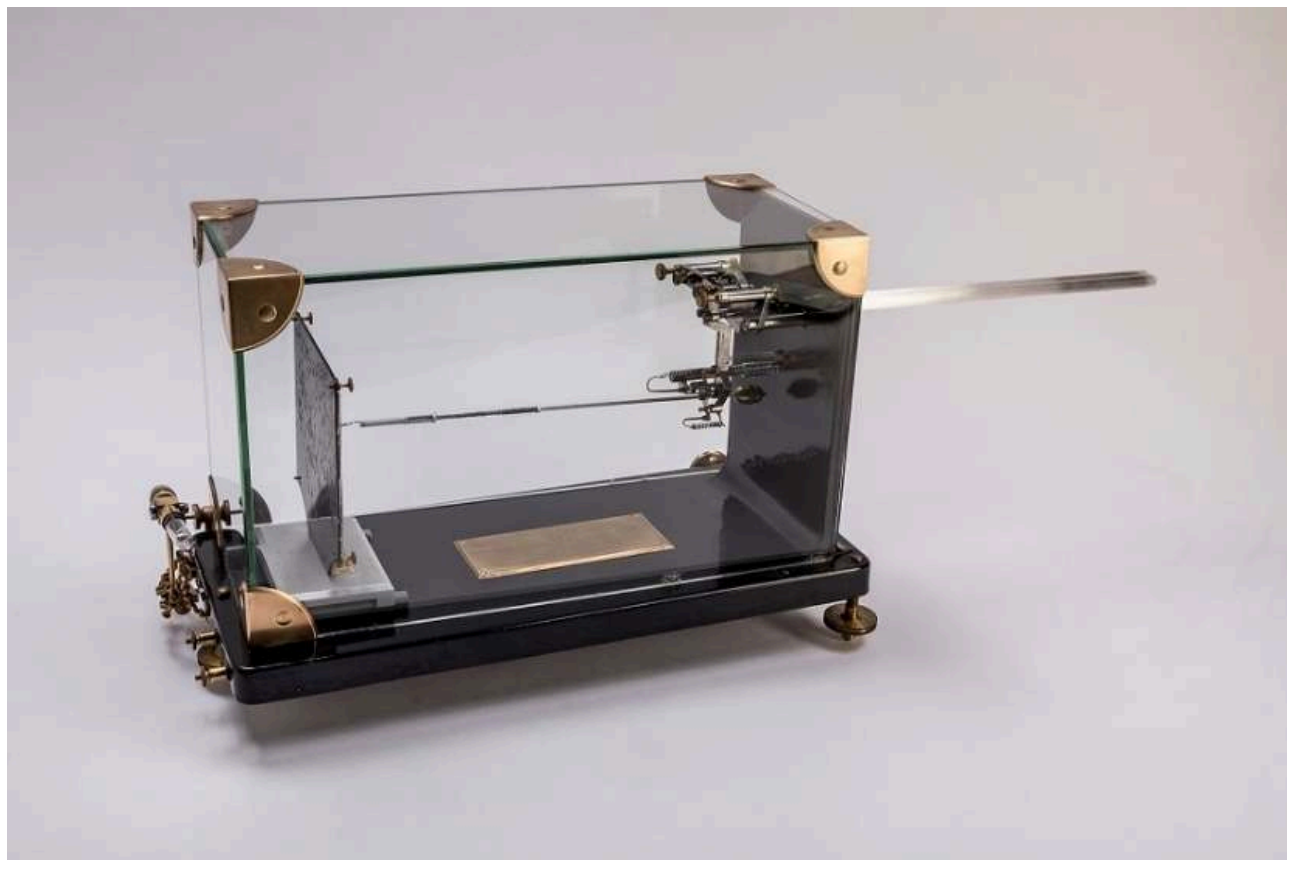

(c) musée de l'Histoire du fer, Jarville-la-Malgrange, cliché Claude Philippot

16 En 2015, le musée a reçu un don d'objets en fer forgé réalisés par un ouvrier forgeron autodidacte, Gabriel Voirin (1899-1963). Passionné par le fer forgé, celui-ci produisait des meubles, des objets décoratifs et utilitaires en fer forgé. Toutes les pièces reçues en don au musée n'ont pas été inscrites à l'inventaire. Le modèle réduit de l'atelier de forgeron est devenu une pièce de collection «musée de France » après avoir reçu l'avis favorable de la commission scientifique régionale de la DRAC. Reproduisant dans le détail et en modèle réduit un atelier complet de forgeron, l'atelier miniature montre les machines et les outils servant au travail du fer: forge, étau, poste de travail à l'enclume, tour à métaux, plusieurs machines à percer, cisaille à métaux, bordeuse, etc. (fig.4). 
Fig.4. L'atelier de forgeron réalisé par Monsieur Voirin, fer forgé, 1920-1930

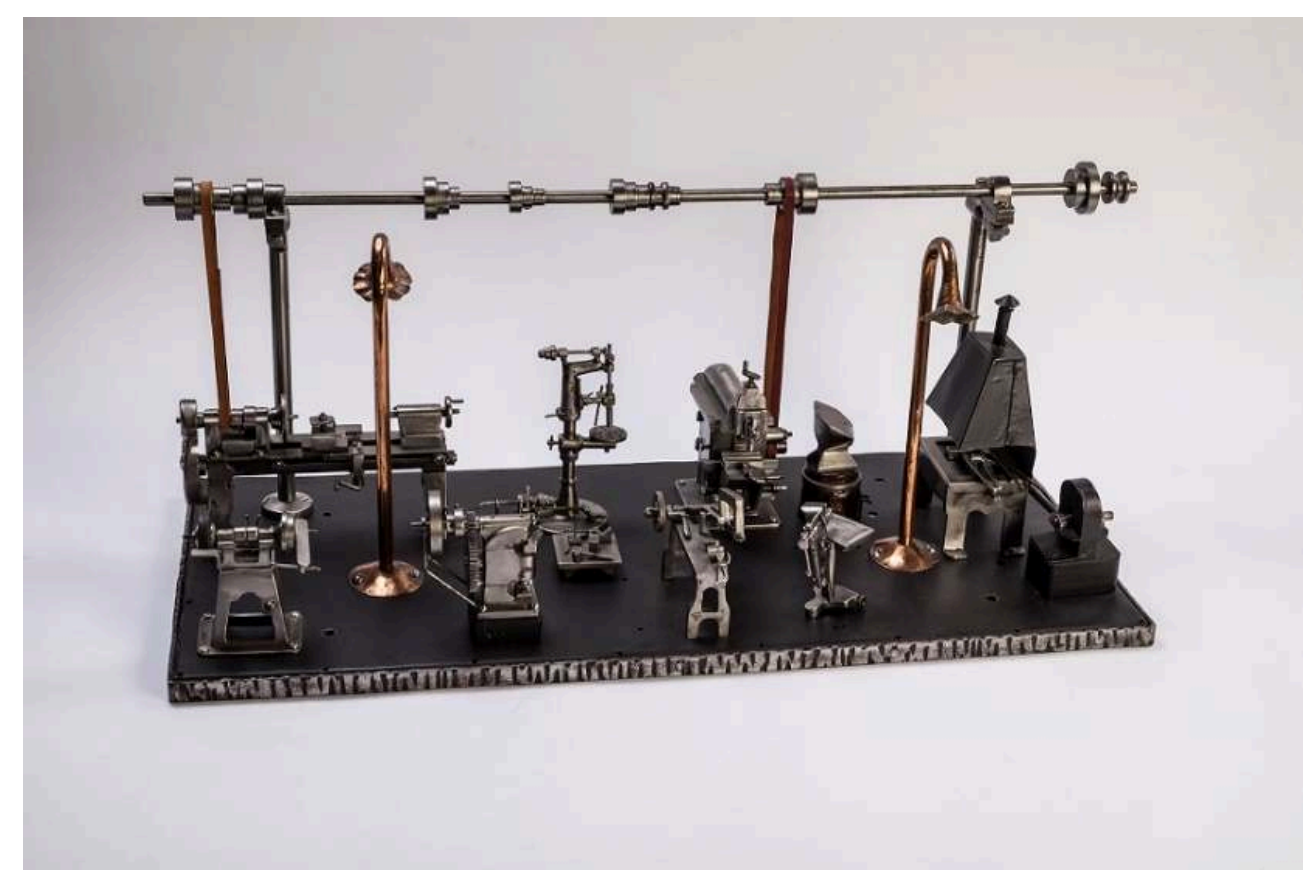

(c) musée de l'Histoire du fer, Jarville-la-Malgrange, cliché Claude Philippot

17 À travers ces deux objets de collections, ce sont bien des parcours de vie, des anecdotes et expériences que le musée peut présenter au public.

\section{L'objet scientifique et technique « polysémique »}

\section{Raconter des histoires qui parlent au public}

Un objet scientifique, technique et industriel est porteur d'histoires. Il doit contribuer à informer les publics, à créer des envies de connaissance et de découverte, mais aussi à susciter des interrogations. Prenons l'exemple de l'escalier de la tour Eiffel, dont un tronçon fait partie de la collection patrimoniale du musée de l'Histoire du fer. Démonté au moment des grands travaux de rénovation entre 1982-1983, ce tronçon a été offert en 1985 au musée par la Société Nouvelle d'Exploitation de la tour Eiffel. Intégrer cet élément dans les collections (malgré les difficultés de conservation) semblait aller de soi : la tour Eiffel est en partie lorraine : le métal qui la compose est un fer puddlé extrait des mines de fer de Ludres, au Sud-ouest de Nancy, transformé en poutrelles de fer puddlé laminé dans les fonderies de Pompey au Nord de Nancy! Qu'un tronçon d'escalier revienne en Lorraine pour y être exposé constitue donc une forme de retour aux sources ${ }^{6}$. Deux films ont été produits par le musée; l'un expose l'histoire industrielle de la construction de la tour Eiffel avec la fabrication des poutrelles métalliques à la Société des Aciéries de Pompey en Lorraine, l'autre suit la restauration de cet objet «hors norme » de la collection: le tronçon de l'escalier de la tour Eiffel mesurant plus de 10 mètres de hauteur et exposé en permanence dans le jardin du musée $^{7}$ (fig.5). 
Fig.5. Tronçon de l'escalier de la Tour Eiffel

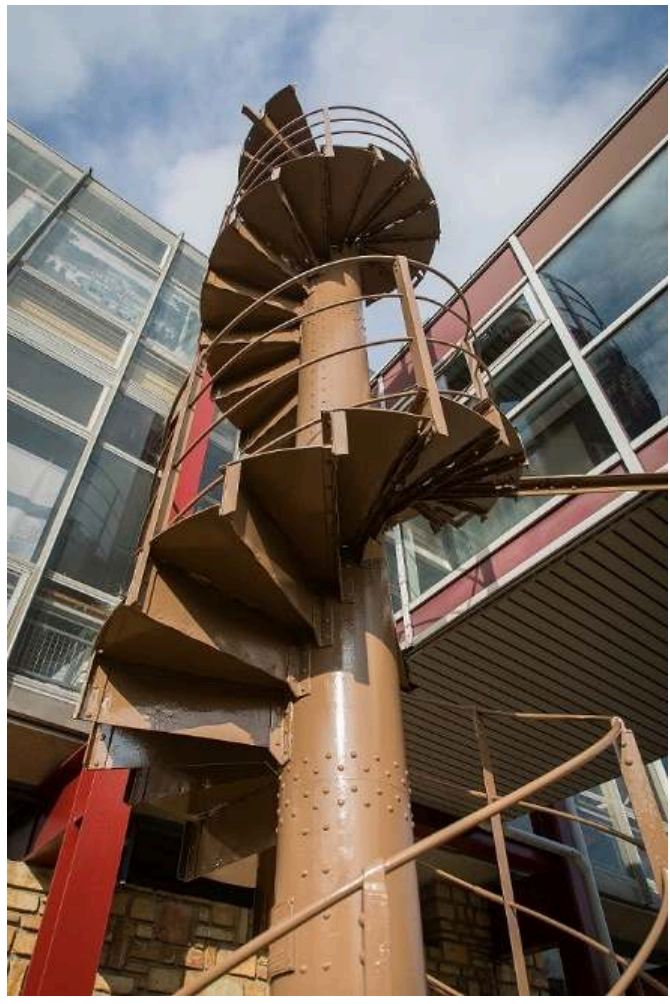

(c) musée de l'Histoire du fer, Jarville-la-Malgrange, cliché Claude Philippot. inv. Nº 1985.3.1

19 Autre exemple d'une œuvre riche de récits : en 2016, le musée a reçu en don de la société Arcelor Mittal France, une immense œuvre de l'artiste mosellan Camille Hilaire (1916-2004). Cette peinture sur toile de 3,29 $\mathrm{m}$ de hauteur et de 7,45 m de longueur intitulée Sidérurgie était présente au moment de sa commande en 1952-53 dans la salle du Conseil d'administration de Sidélor à Paris (Lassère 2021). Dans les années 1960, elle décore le restaurant des ouvriers des Hauts-fourneaux de Knutange, puis les Grands Bureaux de Rombas avant d'orner les Grands Bureaux de Florange jusqu'à l'été 2016, date de son arrivée au musée de l'Histoire du fer (fig.6). Cette œuvre témoigne, en tant qu'œuvre artistique, des grandes commandes des industriels et atteste, comme œuvre documentaire, de l'activité sidérurgique lorraine. Cette œuvre illustre l'industrie pendant les Trente Glorieuses, les fusions des grands groupes sidérurgiques, puis les fermetures et les abandons de sites. Un film, produit par le musée en $2016^{8}$, raconte le démontage de l'œuvre dans les Grands Bureaux de Florange, sa restauration dans l'atelier du restaurateur Christian Vibert et son installation définitive au musée de l'Histoire du fer. 
Fig.6. Camille Hilaire Sidérurgie ou encore Décor des Grands Bureaux de Florange

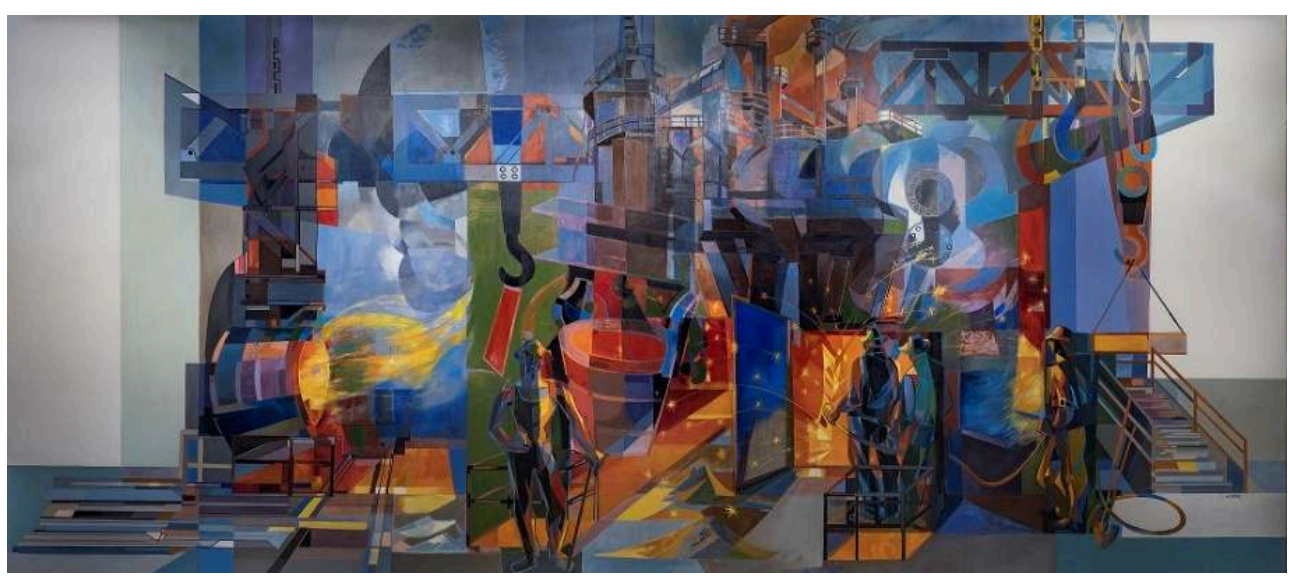

(c) musée de l'Histoire du fer, Jarville-la-Malgrange, cliché Claude Philippot. Inv 2016.1.1

\section{Attention un train peut en cacher un autre ou : des différentes façons de regarder le patrimoine}

La façon dont on regarde un objet de science et de technique peut en modifier le sens. L'exemple des tronçons de l'escalier de la tour Eiffel est à ce titre éloquent. En 1985, outre celui reçu au musée de l'Histoire du fer, deux autres tronçons ont été offerts à des structures culturelles parisiennes : l'un a été offert à Universcience - Cité des Sciences et de l'Industrie et l'autre au musée d'Orsay, musée spécialisé dans les Beaux-arts, l'architecture, les Arts décoratifs du $19^{\mathrm{e}}$ siècle. Cette entrée d'un même objet dans trois collections différentes est porteuse de sens. Au musée de l'Histoire du fer, le sens de ce versement est l'origine lorraine du fer de la tour Eiffel et la prouesse technique de la construction d'une tour de 300 mètres en fer à la fin du $19^{\mathrm{e}}$ siècle. Au musée d'Orsay, il s'agit davantage de valoriser l'architecture du $19^{\mathrm{e}}$ siècle et à Universcience La Villette de valoriser la figure de l'ingénieur; Universcience n'ayant d'ailleurs pas de vocation patrimoniale. Un même objet permet donc trois points de vue différents selon les pratiques professionnelles. Le vrai sujet est ici le regard et le point de vue qui détermine le sens de l'objet technique!

\section{Des regards différenciés pour enrichir les discours sur un objet}

21 Ce qui peut être porteur de sens pour un objet des techniques, c'est d'être présenté selon plusieurs points de vue: objet technique, mais aussi objet de société, objet d'anthropologie, d'industrie, d'art contemporain...

Une exposition permet d'illustrer ce propos : le musée des civilisations de l'Europe et de la Méditerranée (MUCEM) a présenté une exposition sur «Le clou» de novembre 2015 à juin 2016 dans le Centre de Conservation et de Ressources à Marseille. À la question «Pourquoi faire une exposition sur les clous? (Airault 2016), Damien Airault, le commissaire de l'exposition répond: "Le clou regroupe des visions d'artistes, d'archéologues, d'ethnologues et d'anthropologues, mais aussi de psychanalystes ou d'historiens des techniques dans un propos qui font se rejoindre science, art contemporain et artisanat ». Damien Airault interroge plusieurs spécialistes: à un historien de l'art, il demande «Comment envisager la présence du clou dans le champ 
des Beaux-arts? À des archéologues: "Quelle est l'importance du clou et plus généralement des objets métalliques en archéologie?", "Que sait-on de l'apparition des métaux? ", À un anthropologue, il demande de parler d'une forge... Autant de regards qui donnent du sens à un objet $a$ priori très banal.

Au musée de l'Histoire du fer aussi, la variété des regards et des points de vue enrichit les objets des sciences et techniques. Comment cela est-il fait? À quoi cela sert-il ? Comment fait-on fonctionner cette machine? Qui l'a utilisée? Quelle innovation est apportée par cet objet? Autant de questions qui peuvent être abordées dans la scénographie. Lier les collections à des questions de société ou d'actualité, raconter une histoire significative, éclairer les objets d'un regard artistique est essentiel pour présenter de façon attractive ce patrimoine technique et industriel.

24 En juin 2015 ont eu lieu à Nancy les journées «Science and You » de la culture scientifique et technique. À cette occasion, les trois établissements de culture scientifique et technique de la Métropole du Grand Nancy ont organisé un événement original: le Muséum-Aquarium de Nancy, le Jardin botanique Jean-Marie Pelt et le musée de l'Histoire du fer ont tous trois exposé un objet de leurs collections caché à la vue du public. Ce dernier devait découvrir les objets cachés grâce aux seuls commentaires transmis par des experts. En agrégeant les points de vue et discours différents sur un même objet, le public disposait d'indices pour découvrir l'objet caché. Pour le musée de l'Histoire du fer, nous avions choisi comme objet polysémique, le poste de soudure à arc du designer Jean Prouvé (1901-1984), datant de 1926 et commandé auprès de la Société Autogène Française (fig.7). Un premier commentaire émanait du restaurateur de l'objet qui l'avait restauré très récemment. Un deuxième commentaire présentait l'illustre designer à qui a appartenu cette machine d'équipement moderne, enfin le troisième commentaire exposait l'innovation technologique de la soudure: les tôles peuvent être assemblées l'une à l'autre permettant un allègement, une économie pour la production en série et l'apparition d'une architecture métallique innovante. Grâce à cette technique, Jean Prouvé a développé les possibilités infinies d'assemblages entre plusieurs métaux. 
Fig.7. Poste de soudure à arc de Jean Prouvé, SAF, 1926

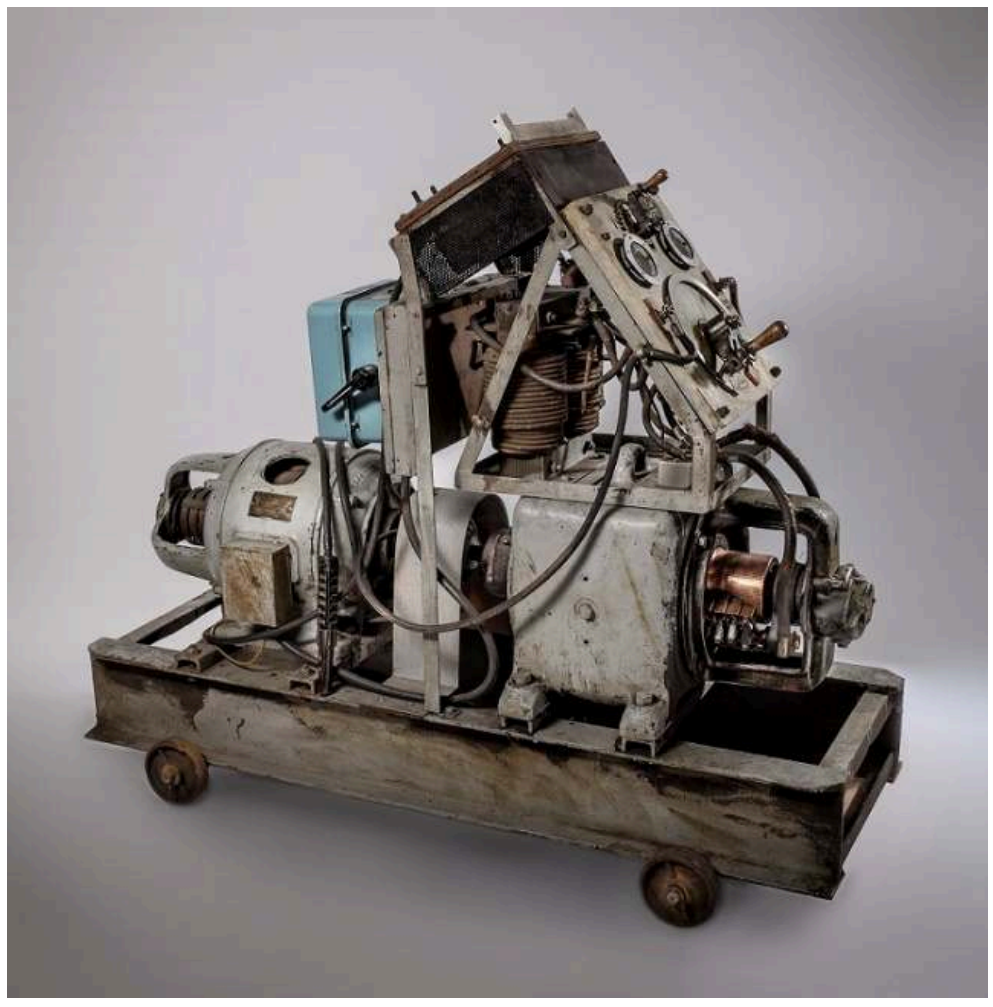

(c) musée de l'histoire du fer, Jarville-la-Malgrange, cliché Claude Philippot, inv. Nº -D.2015.4.1

Ce jeu sous forme d'enquête a rencontré un franc succès. Dévoiler progressivement plusieurs aspects d'un objet permet d'accroître les connaissances et de proposer une démarche itérative au public: l'association des différents discours sous la forme d'un puzzle donne un sens précis à l'objet.

\section{Des regards différenciés à l'international : Élargissons le propos au patrimoine monumental}

Concernant le patrimoine monumental des sciences et des techniques, les regards et les modes de conservation sont différents selon les pays. Prenons l'exemple des hautsfourneaux : plusieurs pays européens, le Luxembourg, l'Allemagne, l'Espagne, la France, se sont posés les mêmes questions quant à ces témoignages représentatifs d'une histoire industrielle et d'une culture commune. Tous ont fait le choix de la conservation d'un ou de plusieurs hauts-fourneaux plutôt que de la démolition.

En France, le parti-pris a été de conserver U4, le dernier des haut-fourneaux du site industriel d'Uckange en Moselle, dans la vallée de la Fensch (fig.8). Uckange est un site industriel créé en 1890, pendant la période de l'Annexion allemande, par des industriels sarrois, les frères Stumm. Le haut-fourneau U4, à l'origine allemand puis devenu français après le Traité de Versailles, a bénéficié d'une rénovation entre 1930 et 1932. La production d'acier y est stoppée en 1991. Classé monument historique depuis 2001, il est le seul haut-fourneau fonctionnant au $20^{\mathrm{e}}$ siècle destiné à être conservé en France. En 2005, après quinze ans d'abandon, la Communauté d'Agglomération du Val de Fensch devient propriétaire du site et entame immédiatement d'imposants travaux de mise en sécurité et de désamiantage, préalable indispensable à l'ouverture du site au 
public en 2007. Une œuvre artistique «Tous les soleils» de l'artiste Claude Lévêque consiste en une mise en lumière et un cheminement visuel du haut-fourneau U4. L'état de conservation d'U4 est au plus près de son état au moment de l'arrêt de la production. C'est cet état de «1991» que les conservateurs des Monuments historiques, les spécialistes de la corrosion des métaux et la Communauté d'Agglomération du Val de Fensch cherchent à conserver durablement, non sans difficulté car l'édifice se dégrade inexorablement (Tornatore 2010).

Fig.8. Le haut-fourneau au sein du Parc du haut-fourneau U4, à Uckange en Moselle

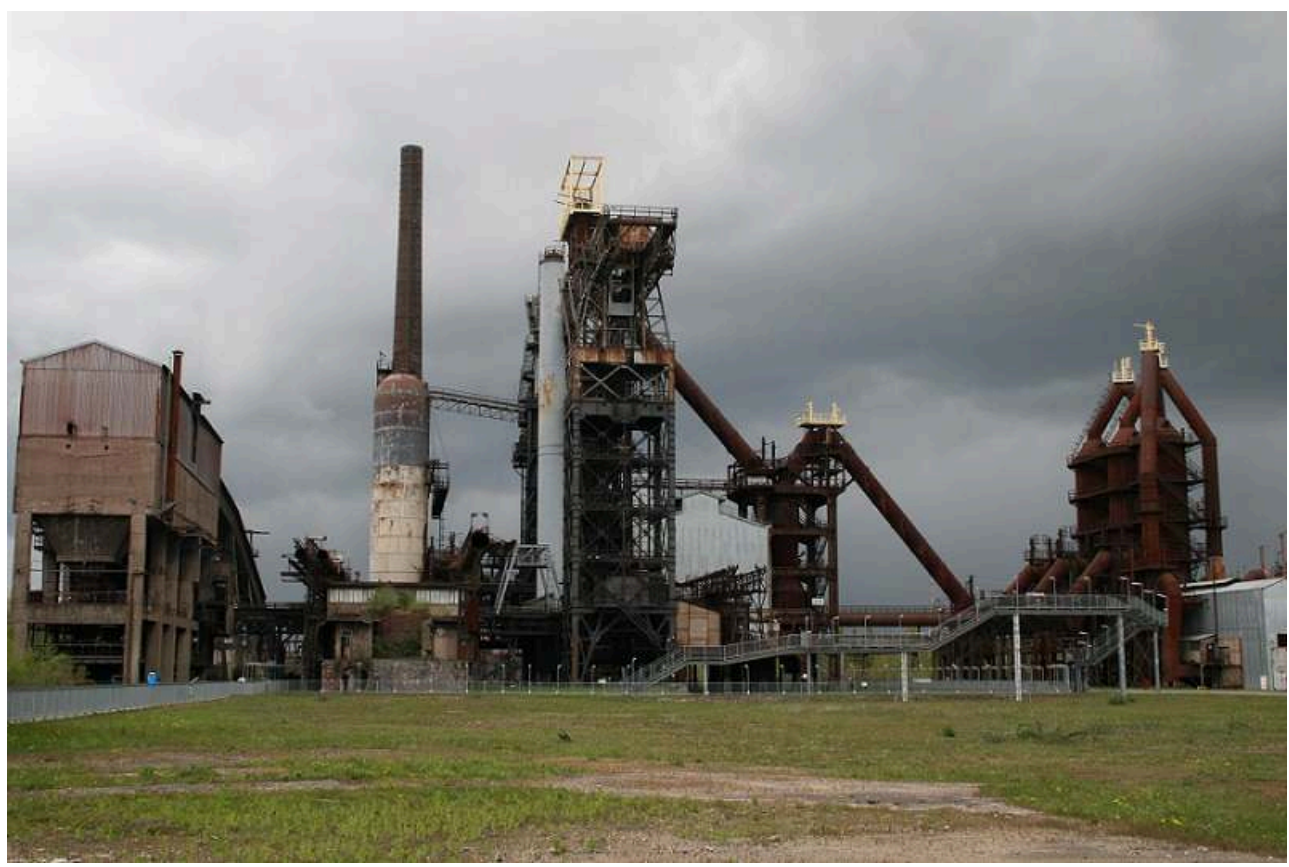

(c) cliché Odile Lassère

En Allemagne, le site de Völklingen dans la Sarre a produit de l'acier de 1873 à 1986 . Il est classé «Patrimoine mondial de l'UNESCO » depuis 1994. Il constitue le seul site sidérurgique au monde destiné à être conservé dans son intégralité (fig.10). Il s'agit ici de maintenir l'aspect authentique du site avec le minimum de modifications nécessaires pour assurer la sécurité des visiteurs. Les différentes parties de l'usine sont régulièrement entretenues et repeintes de couleur rouille et la visserie est renouvelée autant que nécessaire. 
Fig.9. Usine sidérurgique de Völklingen

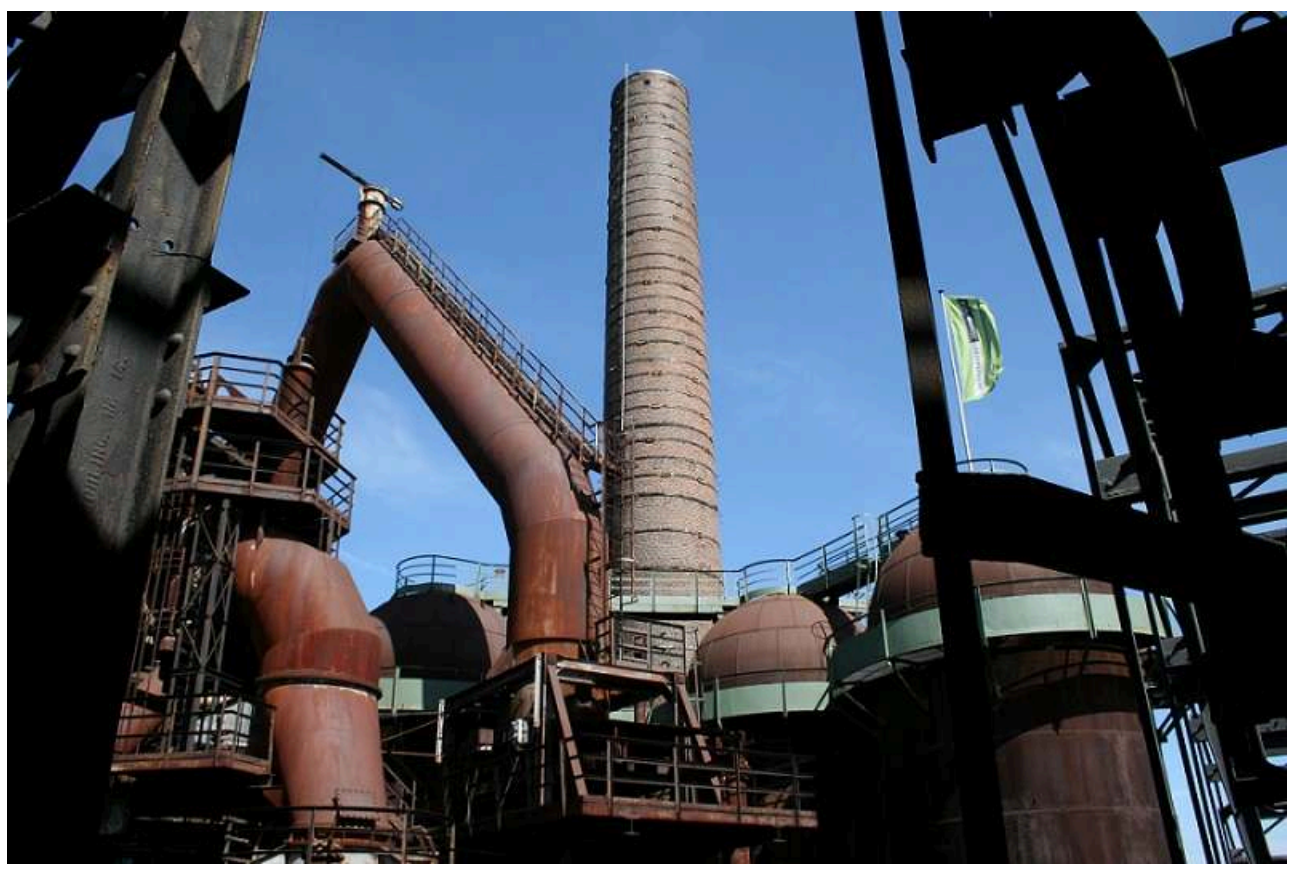

Ce complexe sidérurgique de la Sarre a fonctionné de 1873 à 1986. II est devenu patrimoine mondial de l'Unesco, en 1994.

(c) musée de l'Histoire du fer, Jarville-la-Malgrange, cliché Odile Lassère l'acier jusqu'en 1984. Ce haut-fourneau a, depuis, fait l'objet d'une rénovation complète et se présente aujourd'hui sous un nouvel aspect lui assurant une protection optimale (Bernabé, Salom, Albertos Puebla et Castello 1997). Les surfaces métalliques du hautfourneau ont été entièrement sablées avant une mise en peinture avec des résines à base de polyuréthane. L'aspect n'est donc plus celui d'origine; le haut-fourneau se présente aujourd'hui sous le visage d'un site industriel idéalisé et entièrement repeint.

Enfin, deux hauts-fourneaux du site de Belval, à Esch-sur-Alzette, au Luxembourg sont conservés, entièrement repeints. Ce site de Belval, producteur d'acier de 1872 à 1985, est aujourd'hui pleinement intégré dans une nouvelle zone urbaine comprenant université, forum, entreprises, zone commerciale.

Ces exemples illustrent les différences de stratégie en matière de conservation en Europe. Sauvegardés durant les mêmes périodes, entre 1995 et 2005, ces hautsfourneaux ont subi des choix de conservation, de rénovation, de réutilisation et de mise en valeur différents selon les traditions, les règlementations et les principes déontologiques de conservation du patrimoine de chacun des pays. Une des difficultés majeures de la conservation de ce type de patrimoine technique et industriel est dans sa monumentalité : le gigantisme est un sérieux handicap pour la conservation. En effet, si le musée scientifique et technique a facilement vocation à collecter les machines, il n'en a malheureusement pas les moyens quand il s'agit de machines ou d'objets de très grandes dimensions, faute d'espace adapté. C'est pourquoi trente ans après leur sauvegarde, ni le musée d'Orsay ni la Villette ne présentent leurs propres tronçons de l'escalier de la tour Eiffel, d'où l'extrême intérêt de poursuivre cette présentation au musée de l'Histoire du fer! 


\section{Comment valoriser l'objet scientifique et technique?}

32 Tous les objets ou éléments de patrimoine précédemment évoqués ont-ils encore du sens aujourd'hui ? Comment faire venir au musée de l'Histoire du fer le public alors que ce dernier n'a plus dans son environnement autant de repères de l'industrie sidérurgique qu'en 1966? Les cheminées d'usines, hauts-fourneaux, tours cowpers (installations annexes des hauts-fourneaux), quais de déchargement, trains de marchandises ont disparu mais notre paysage familier reste empreint de vestiges et de nouvelles déclinaisons industrielles et technologiques... Ne faudrait-il pas redonner à comprendre le sens technique des objets? Ne faudrait-il pas actualiser le discours et conduire le public à s'interroger sur le monde industriel d'aujourd'hui, notre rapport à l'innovation technologique, à la pollution, au développement durable, aux nouveaux matériaux... C'est dans ce sens que nous bâtissons le Projet scientifique et culturel du musée pour un musée renouvelé, un lieu de connaissance, d'éducation et de plaisir. Un musée est « une collection permanente composée de biens dont la conservation et la présentation revêtent un intérêt public et organisée en vue de la connaissance, de l'éducation et du plaisir du public ${ }^{9}$ ".

Voici quelques pistes de réflexion transcrite dans le Projet Scientifique et Culturel du musée de l'Histoire du fer.

\section{Rattacher l'objet patrimonial à son territoire}

Expliquer le sens technique mais aussi les points de vue historiques, sociaux, artistiques des collections constitue un enjeu pour le musée. Autre enjeu : relier le musée à son territoire. Implanté à proximité de Nancy, ce musée est une création ex nihilo issue du colloque universitaire et scientifique de 1955 : «Le fer à travers les âges » et du centre de recherche en histoire de la sidérurgie et souhaitée par ses fondateurs Édouard Salin, Albert France-Lanord et Bertrand Gille. Son ancrage en Lorraine, au cœur d'une région riche en minerai de fer et en concentration d'industries sidérurgiques, est déterminant. Toutes les collections du musée peuvent être reliées au territoire de la région Grand Est. Rapprocher le patrimoine du territoire permet de le rendre concret et accessible. C'est un axe à privilégier et à développer pour une plus grande lisibilité et proximité des collections avec le public. À propos de Maurice Daumas (1910-1984), pionnier de l'histoire des techniques et du patrimoine industriel, directeur du musée des arts et métiers dans les années 1960-1970 mais aussi promoteur du musée de l'Histoire du fer à ses débuts, Denis Woronoff, historien spécialiste de l'histoire économique de la France, écrit : «Il a montré à quel point l'histoire des techniques gagnait en lisibilité et en densité lorsqu'on l'ancrait sur un territoire, lorsqu'on l'étudiait dans ses conditions concrètes d'effectuation, de pratiques, et non plus dans un système plus intellectuel d'une histoire un peu virtuelle. » (Woronoff 2014) ${ }^{10}$.

Aussi un des axes retenus consiste à valoriser le patrimoine scientifique et technique dans son lien avec le territoire et l'histoire locale. Le "Parcours d'En Fer", scénographie de douze objets, réalisée en 2016 au musée de l'Histoire du fer, a mis en valeur un coq en tôles d'acier rivées et soudées (fig.10). Ce coq, réalisé par des ouvriers sidérurgistes de Longwy en grève en 1979, a été acheté par le musée en 2005. 
Fig.10. Coq des sidérurgistes en grève, tôle de fer soudée

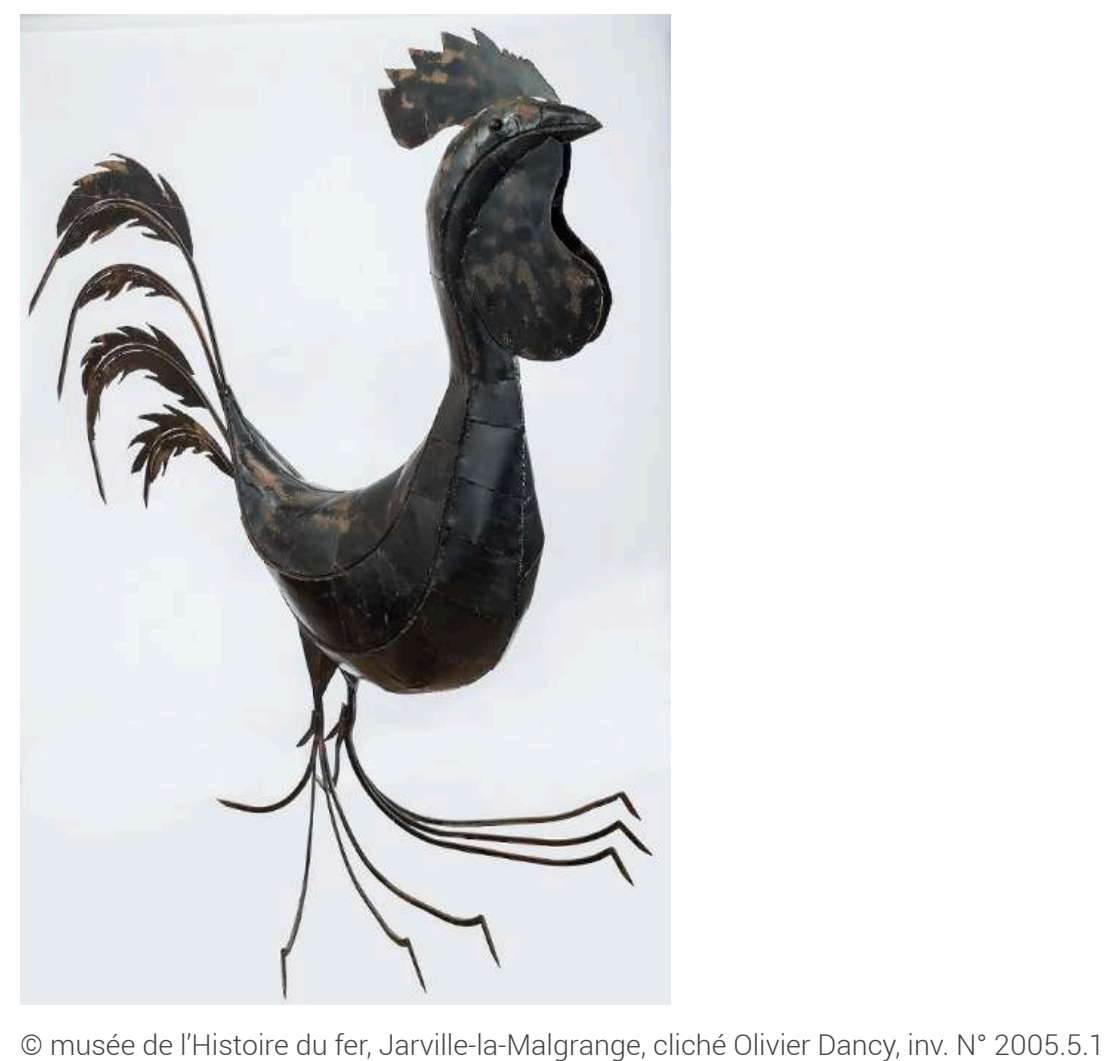

Longwy a été en effet le théâtre de mouvements sociaux de grande ampleur au moment des difficultés de la sidérurgie lorraine. Deux plans de restructuration successifs, en 1978 et en 1984, mis en œuvre à Longwy, ont supprimé des milliers d'emplois. La mobilisation, portée notamment par la CGT, a été d'autant plus vive que Longwy est une cité vivant de la sidérurgie depuis la fin du $20^{e}$ siècle. Dans les années 1970 , la sidérurgie française, endettée, tarde à moderniser ses installations. De nouveaux procédés, notamment l'aciérie à l'oxygène, rendent le haut fourneau moins indispensable. À partir des années 1970, la sidérurgie française se relocalise à Fos-surMer et à Dunkerque. Aujourd'hui, la part de la Lorraine dans la production mondiale d'acier est marginale. La sidérurgie lorraine emploie désormais un peu plus de 4000 personnes (toutes catégories salariales confondues) alors qu'elle faisait travailler environ 80000 ouvriers dans les années 1960 (Raggi 2019).

Les grandes grèves de Longwy ont symbolisé le repli de cette industrie régionale sur certains sites, certes performants mais très circonscrits. Le coq élaboré par les sidérurgistes de Longwy symbolise la lutte menée par les «hommes du fer » de la région Lorraine pour sauvegarder leurs emplois. Mis en valeur avec des extraits de reportages montrant les cortèges des grévistes, le coq du musée présenté dans son contexte territorial et historique est à lui seul, une leçon d'histoire. Toujours en cours de réflexion et de modification, le parcours permanent du musée de l'Histoire du fer est revu dans une scénographie adaptée et modernisée. Ce nouveau parcours permanent intitulé «La fabrique des m(atéri)étaux » permettra de faire le lien entre les métaux et les matériaux en ouvrant sur la recherche-développement de matériaux performants pour les nouveaux usages. Ce nouveau parcours permanent s'ouvrira pour le public en 
2022 par ce "coq des sidérurgistes de Longwy", symbolisant à la fois l'épopée industrielle et le travail du métal.

\section{Faire participer le public}

Si la mission du musée de l'Histoire du fer reste la transmission des savoirs scientifiques et techniques, il paraît souhaitable de trouver de nouvelles formules pour rendre le visiteur actif, et ce dès le plus jeune âge. Extraire certains objets du parcours, ou bien identifier des thématiques, les mettre au centre d'un atelier participatif avec le public permettrait de repenser des présentations: nous pourrions ainsi créer un parcours participatif sur le modèle des Muséomix ${ }^{11}$, ces ateliers qui permettent à une communauté d'investir le musée pendant trois jours et de réinventer, de réinterpréter certains objets du parcours. Cette expérience Muséomix menée dans des musées de sciences et de techniques (comme au Musée d'art et d'industrie de Saint-Etienne en 2014 ou encore dans les musées de la gorge d'Ironbridge dans le Shropshire en Angleterre en 2013) est d'autant plus porteuse qu'elle offre la possibilité à une communauté de médiateurs, designers, développeurs, graphistes, communicants, scientifiques, de s'approprier le musée pendant un marathon créatif de trois jours. Muséomix est un événement international. Chaque année en novembre, les participants investissent plusieurs musées dans le monde.

Pour le musée de l'Histoire du fer, imaginons comment proposer une autre expérience de visite, plus interactive, et des outils numériques facilitant la découverte des collections.

\section{Intégrer le patrimoine scientifique et technique contemporain}

Que signifie intégrer le contemporain pour un musée des sciences et techniques? D'où vient cette préoccupation? Est-ce acquérir un patrimoine récent ou porter un regard d'aujourd'hui sur le passé ? Quel rôle doit avoir le musée ? Comment la collecte peutelle être mise en forme pour le contemporain?

41 L'idée est d'établir des liens entre le musée et la société d'aujourd'hui selon trois grands axes :

42 En premier lieu, le souci du contemporain pour un musée envisagé comme un lieu vivant, un foyer de création et un outil de développement. Il s'agit moins ici d'une collecte d'objets et de collections que de renouveler les thèmes abordés, les modes de présentation, les activités ou les pratiques: le musée devient un lieu de vie et de création. Le musée de l'Histoire du fer a ainsi servi, à plusieurs reprises, de lieu vivant de connaissance sur les métaux et les matériaux et a permis des rencontres: les étudiants de la section design de l'École Nationale Supérieure d'Art et de Design de Nancy ont rencontré les élèves du BTS conception de produits et du BTS fonderie du Lycée Henri Loritz de Nancy. Ensemble, ils ont créé des objets de design en fonderie. Le musée permet d'exposer la méthode de production, la technique de la fonderie au sable et le résultat de ces créations.

43 En deuxième lieu, le musée doit se transformer pour refléter le monde actuel. Depuis quelques années, le musée développe une programmation culturelle de qualité offrant une tribune à la recherche en cours. Chaque année, à l'occasion de la journée des droits de la femme, le 8 mars, ou encore à l'occasion de la fête du travail, le $1^{\mathrm{er}}$ mai, des 
chercheurs, des passionnés ou des membres d'associations viennent rencontrer le public pour présenter des sujets offrant débats : par exemple, nous avons pu aborder au musée la place de la femme dans l'industrie, les mathématiques et les femmes à travers le portrait de Sophie Germain, mathématicienne du début du $19^{\mathrm{e}}$ siècle, la bataille de Florange et les récits sur les grands mouvements de grève dans la sidérurgie à la fin du $20^{\mathrm{e}}$ siècle et au début du $21^{\mathrm{e}}$ siècle.

Enfin, en troisième lieu, le musée doit se renouveler en intégrant des objets contemporains. Prescripteur du patrimoine contemporain, le musée pourrait lancer une collecte participative mais aussi un recueil de mémoire orale ou des ateliers d'écriture. Pour la période la plus récente, les cinquante dernières années, la logique d'acquisition des «musées de France » retient fréquemment le passage par un stade de " matériel d'étude » (ainsi que défini dans la première partie de cet article), permettant de sélectionner les objets qui pourront entrer ultérieurement dans les collections patrimoniales, grâce au recul de la recherche. La mission PATSTEC, programme de sauvegarde du patrimoine scientifique et technique contemporain (Cuenca 2016) a été créée en 2003, pour sauvegarder des témoignages de la recherche scientifique en France depuis la Seconde guerre mondiale ${ }^{12}$. Cette mission a été confiée en 2003 par le ministère de l'Enseignement supérieur et de la Recherche au musée des Arts et Métiers.

Cette collecte du contemporain incite à se poser des questions sur les savoirs à conserver, les domaines scientifiques actuels qui peuvent être considérés comme majeurs, les instruments à préserver, pour refléter le dynamisme de la recherche contemporaine des $20^{\mathrm{e}}$ et $21^{\mathrm{e}}$ siècles.

Pour le patrimoine contemporain, voici les critères retenus: l'intérêt scientifique, l'intérêt historique, la rareté (maquette, prototype, modèle, série de référence) par rapport à un fabricant, une évolution technologique marquante, une diversité des formes, un design et le critère symbolique, les témoignages liés. Aujourd'hui, cette mission PATSTEC compte plus de 18000 objets et 60000 médias attachés à ces objets avec une base de données en ligne ${ }^{13}$. La collecte concerne toutes les traces matérielles et immatérielles, qui « font savoir » et « font comprendre » la recherche et les avancées technologiques de ces soixante dernières années. En raison de l'ampleur des sciences et des techniques et des objets patrimoniaux créés depuis la Seconde guerre mondiale, la conservation d'un tel patrimoine ne relève-t-elle pas de la «mission impossible»? Pour l'instant, la mission PATSTEC n'a pas de vocation patrimoniale. Elle ne s'inscrit pas dans le formalisme de la loi musées : c'est une mission qui intervient dans d'autres sphères administratives que celle des musées de France et qui met en œuvre d'autres pratiques professionnelles.

47 La démarche est néanmoins très intéressante : comment relier cette mission PATSTEC à l'histoire des sciences et techniques? Quel est l'avenir de la mission? «La fabrique du patrimoine ", au sens de distinction d'objets patrimoniaux, n'est-elle pas réservée exclusivement aux institutions culturelles? En résumé, que vont devenir les objets identifiés et collectés ? La Métropole du Grand Nancy avec l'Université de Lorraine s'est emparée de cette mission en 2020. Une chargée de mission inventorie le patrimoine scientifique, technique et industriel contemporain des laboratoires publics et des centres de recherche et développement privés notamment dans le domaine des matériaux musée de l'Histoire du fer avec la mission PATSTEC est une proposition pour valoriser 
l'innovation. Lier en effet ensemble une collection patrimoniale, une collection "matériel d'étude», mais aussi le patrimoine contemporain, les laboratoires de recherche, les industriels, a du sens. Sur l'aspect social, le musée peut être l'initiateur d'une enquête-collecte sur l'histoire sociale et les liens que nous entretenons avec les matériaux et les territoires de l'industrie en Lorraine.

\section{Conclusion}

49 À l'ère du tout numérique, le musée de l'Histoire du fer doit continuer à privilégier la rencontre avec l'objet sous ses différents aspects. Penser l'établissement comme un musée de Société décloisonné, qui aide à comprendre le monde, est également une priorité. Le PSC du musée est aujourd'hui encore à préciser dans le respect du Code du Patrimoine et du projet culturel métropolitain.

Mon point de vue est le suivant: les collections sont le fondement du musée qui doit être un lieu vivant, jouant de la complémentarité des collections entre elles, mettant en valeur œuvres et objets qui racontent des histoires : objets du contemporain, objets relatifs aux pratiques sociales, liens entre culture et sciences... Décloisonner les collections pour privilégier des thématiques, telle pourrait être la ligne directrice d'une refonte complète du musée en plusieurs parcours complémentaires: un parcours historique sur l'histoire de l'industrie de la région Grand Est (Lassère 2016), un parcours sur l'histoire des techniques et des matériaux sous le double aspect de la fabrication, mais aussi des usages et des fonctions des objets, un parcours spécifiquement dédié aux enfants avec des expériences et des manipulations et, cerise sur le gâteau, aspect très original pour un musée des sciences et techniques: un parcours Beaux-arts qui donnerait à voir et à comprendre les gestes du travail et les paysages de l'industrie.

51 Ce cheminement Beaux-arts atypique couvrirait une longue période, depuis les gravures du $17^{\mathrm{e}}$ et $18^{\mathrm{e}}$ siècle jusqu'aux œuvres d'art ou au design contemporains. L'exemple lyonnais du musée des Confluences peut ici aider à la réflexion. Ses collections sont issues de quatre institutions : le muséum d'histoire naturelle de Lyon (1772-2007), le musée Guimet de Lyon (1879-1889, 1913-1978), le musée colonial (1927-1968) et les collections de l'œuvre de la Propagation de la foi. Il a pour objectif de rendre compte des rapports entre les sciences et les sociétés en insistant sur la pluralité des uns et la diversité des autres. En organisant la collection en quatre parcours permanents: "Origines, les récits du monde», "Espèces, la maille du vivant », "Sociétés, le théâtre des hommes ", "Éternités, visions de l'au-delà », le musée a choisi de décloisonner les collections et de privilégier des thématiques. Dans l'attente d'une rénovation complète, la programmation d'expositions temporaires au musée de l'Histoire du fer peut permettre d'explorer ces différentes pistes.

Intégrer le musée dans un réseau international avec la Sarre et le Luxembourg est un autre axe du PSC. Le musée doit développer, avec ses voisins, une culture commune autour de formations, d'événements coproduits, de confrontations de collections. Renforcer les liens avec l'Université de Lorraine constitue également un axe à encourager car le musée, lieu de délectation pour le public, est aussi un lieu d'apprentissage, de confrontation, d'ouverture à l'esprit critique et de participation autour de collections et d'idées... autant de propositions pour renouveler le point de vue sur la conservation et la valorisation des objets scientifiques et techniques. 


\section{BIBLIOGRAPHIE}

AIRAULT Damien et alii, «Le clou », MuCEM, FRAC Provence-Alpes-Côte d'Azur, Marseille, Immixtuon Books, éditions Rond-point Projects, 2016

BERNABÉ José M., SALOM Julia, ALBERTOS PUEBLA Juan M. CASTELLO Vincent, « Un cas de reconversion réussie : la sidérurgie des Sagunto (Espagne) », Méditerranée, tome 87, n 3-4, 1997, p. 63-72

CUENCA Catherine, « Le patrimoine scientifique et technique contemporain, histoire d'une sauvegarde et mise en valeur ", in BALLE Catherine, CHAMBAUD Serge, CUENCA Catherine, HALLEUX Robert, THOULOUZE Daniel et alii (dir.), Patrimoine contemporain des sciences et techniques, Paris, La documentation Française, 2016, p. 57-71

CUENCA Catherine, « Sauvegarder le patrimoine scientifique et technique », e-Phaïstos [En ligne], VII-2, 2019, mis en ligne le 15 octobre 2019 ; DOI : https://doi.org/10.4000/ephaistos.4978

DUFFAUT François, «La métallurgie de précision de Pierre Chevenard », Marteau Pilon, t. 24, 2012, p. 35-44

DUFFAUT François, « Pierre Chevenard ou la recherche au cœur de l'entreprise moderne », Entreprises et histoire, $\mathrm{n}^{\circ}$ 83, juin 2016, p. 64-78

FOLZAN Michel, «Le Laboratoire d'archéologie des métaux de Jarville-la-Malgrange, du temps et de la matière ", Musées et collections publiques de France, $\mathrm{n}^{\circ}$ 262, 2011, p. 17-21

FRANCE-LANORD Albert, « Tour Eiffel, fer lorrain », catalogue de l'exposition présentée au Musée de l'Histoire du fer de Jarville-la-Malgrange du 21 juin au 30 septembre 1980

GILLOT Dominique (dir.), Stratégie nationale de culture scientifique technique et industrielle, ministère de l'Éducation nationale, de l'enseignement supérieur et de la recherche, Paris, ministère de la Culture et de la Communication, 2017

JOLY Marie-Hélène, « Le Projet scientifique et culturel a-t-il de l'avenir ? », La Lettre de l'OCIM, 124, juillet-août 2009

JOLY Marie-Hélène, « Le Projet scientifique et culturel aujourd'hui » », La Lettre de l'OCIM, 167, septembre-octobre 2016, p. 40-42

LASSÈRE Odile, « Comment l'industrie vient au patrimoine ? ", dans BALLE Catherine, CHAMBAUD Serge, CUENCA Catherine, HALLEUX Robert, THOULOUZE Daniel et alii (dir.), Patrimoine contemporain des sciences et des techniques, Paris, La Documentation française, 2016, p. $277-288$

LASSÈRE Odile, « Le musée de l'Histoire du fer de Jarville-la-Malgrange fête ses 50 ans... et prépare les cinquante prochains ", Patrimoine industriel, $n^{\circ}$ 68, juin 2016, p. 34-43

LASSÈRE Odile, « Pour un musée des techniques de demain : quelle recette antirouille pour le musée de l'Histoire du fer?», OCIM, 2017

LASSÈRE Odile, « La peinture industrielle de Camille Hilaire, 1952-1970 », dans L’Esthétique des Trente Glorieuses, in ROT Gwenaëlle et VATIN François (dir.), Paris, Éditions Librairie des musées, 2021, p. 172-181

MARIANI-DUCRAY Francine, « 30 ans de patrimoine industriel en France », l'Archéologie industrielle en France, $\mathrm{n}^{\circ}$ 45, décembre 2004, p. 11-13 
PASSAQUI Jean-Philippe, «Textes choisis présentant la démarche scientifique de Pierre Chevenard ", Marteau Pilon, t. XXI, 2010, p. 67-88

RAGGI Pascal, La désindustrialisation de la Lorraine du fer, Paris, Éditions Classiques Garnier, 2019

ROZAY Anne-Sophie (dir.), La sauvegarde du patrimoine scientifique, e-Phaïstos [En ligne], VII-2 | 2019, mis en ligne le 15 octobre 2019 ; https://doi.org/10.4000/ephaistos.4716

TORNATORE Jean-Louis (dir.), L'invention de la Lorraine industrielle. Quêtes de reconnaissance, politiques de la mémoire, Paris, Riveneuve, coll. « Actes académiques », 2010

TORNATORE Jean-Louis (dir.), Le patrimoine comme expérience. Implications anthropologiques, Paris, Maison des Sciences de l'Homme, coll. « Ethnologie de la France et des mondes contemporains », 2019

WORONOFF Denis, « Patrimoine industriel et recherche historique », in BERGERON Andrée, CAILLET Élisabeth, FERRIOT Dominique, GUYON Etienne, GUILLET Philippe, GUIRAUDON, JeanClaude, MAITTE Bernard, MORAND Olivier, VAN PRAËT Michel, Une mémoire pour demain : 30 ans de culture scientifique, technique et industrielle en France, Paris, L'Harmattan, 2014, p. 114-121

\section{NOTES}

1. Se référer aux conférences mélanges en l'honneur de Bertrand Gille, Archives nationales du monde du travail; https://archives-nationales-travail.culture.gouv.fr/Decouvrir/Evenements/ Conferences-Melanges-en-l-honneur-de-Bertrand-Gille, mise en ligne en octobre 2021.

2. Le PSC est à nouveau en discussion après la mutualisation des services des musées au sein du Pôle muséal de la Métropole du Grand Nancy en 2019 et après le changement de gouvernance de la Métropole en 2020.

3. Code du patrimoine et autres textes relatifs aux biens culturels, commenté par Marie CORNU et Vincent NEGRI, éditions Lexis Nexis, 2012.

4. La France est un pays de tradition. La Loi du 4 janvier 2002 codifiée à l'article 451-5 du Code du Patrimoine affirme que « les biens constituant les collections des musées de France appartenant à une personne publique font partie de leur domaine public et sont, à ce titre, inaliénables ».

5. Note circulaire du 19 juillet 2012 relative à la problématique des matériels d'étude et à la méthodologie préalable à l'affectation de certains de ces biens aux collections des musées de France.

6. Se référer à l'exposition Tour Eiffel, made in Lorraine présentée en 2018 au musée de l'Histoire du fer.

7. Chaîne Youtube du Musée de l'Histoire du fer : deux vidéos, «L'histoire de la construction de la Tour Eiffel, avec des images d'archives des collections du Musée », 2018 ; «l'escalier de la tour Eiffel, la restauration!", 2018.

8. Chaîne Youtube du Musée de l'Histoire du fer: vidéo, "Le décor des Grands Bureaux de Florange, Sidérurgie, par Camille Hilaire », octobre 2016.

9. Au titre de l'Art. L410-1 du Code du patrimoine (Livre IV, titre I).

10. On peut aussi se référer aux travaux de Jean-Louis TORNATORE sur la notion de patrimoine (Tornatore 2019).

11. Site internet : www.museomix.org.

12. Voir aussi Catherine Cuenca, «Sauvegarder le patrimoine scientifique et technique » (Rozay 2019).

13. Site internet : www.patstec.fr. 


\section{RÉSUMÉS}

S'appuyant sur des expériences concrètes, l'article interroge les notions de conservation et de mise en valeur du patrimoine matériel scientifique, technique et industriel au sein d'un musée français. Dans une première partie, l'article aborde l'application du Code du Patrimoine (2002) à partir de l'exemple du Musée de l'histoire du fer à Nancy et de ses collections «musée de France ». La deuxième partie présente les spécificités des objets techniques et industriels mais aussi du patrimoine monumental ou immatériel: variété, diversité, polysémie mais aussi difficulté de conservation d'objets composites ou encore monumentaux. Enfin la troisième partie aborde la nécessité de rapprocher ces objets du public : liens soulignés entre les collections et les territoires, présentations scénographiques novatrices, ateliers participatifs impliquant le public. La conclusion ouvre des perspectives pour la valorisation des collections.

Based on concrete experiences, the article questions the notions of conservation of tangible and intangible industrial cultural heritage in a French museum. In the first part, the article addresses the application of the law on heritage in France through the example of the Museum of the History of Iron in Nancy and its museum collection. The second part presents the specificities of the collection of technical and industrial museums but also of monumental or intangible cultural heritage: variety, diversity and difficulty of conservation of composite materials or monumental heritage. Finally, the third part addresses the need to bring these collections closer to the public: links between collections and local communities, innovative exhibitions, digital transformation of the museum, virtual experiences, possibilities for interaction and engagement with the public, workshops involving the public.... The conclusion opens up perspectives that enhance the museum experience.

\section{INDEX}

Mots-clés : histoire des techniques, musée, patrimoine technique, patrimoine scientifique, conservation, valorisation

Keywords : history of technology, museum, industrial heritage, cultural heritage, museum experience

\section{AUTEUR}

\section{ODILE LASSÈRE}

Odile Lassère est conservateur en chef du patrimoine, directrice du musée de l'Histoire du fer de Jarville-la-Malgrange de 2012 à 2019 et directrice des collections, des partenariats et de la recherche au sein du pôle muséal de la Métropole du Grand Nancy depuis 2019. Au Département du Calvados, de 1994 à 1999, elle a géré plusieurs musées et a conduit la rénovation du musée de la Mine du Molay-Littry. De 2000 à 2004, elle a dirigé le musée des automates mécaniques de Neuilly sur Seine puis, de 2004 à 2012, le service études et développement du patrimoine du Département de Seine-et-Marne. Odile Lassère est spécialiste du patrimoine scientifique, technique et industriel. Elle est commissaire de plusieurs expositions temporaires sur l'histoire, l'histoire industrielle, l'architecture métallique, la tour Eiffel. Depuis 2014, elle enseigne la connaissance et la protection du patrimoine scientifique, technique et industriel dans le cadre du Master TPTI (Techniques, Patrimoines, Territoires de l'Industrie) de l'Université Paris 1 
Panthéon-Sorbonne. Elle est membre du conseil d'administration du CILAC, comité d'information et de liaison pour l'archéologie, l'étude et la mise en valeur du patrimoine industriel. Elle est également membre de la Commission scientifique d'acquisition de la DRAC Hauts-de-France. 